\title{
An Applied Equation Inter-Relating Stewart's Parameter and Non- Respiratory Hydrogen Ion Concentration in Arterial Blood Gas
}

\author{
Dr. T. Rajini Samuel M.D* \\ *Assistant Professor of Biochemistry, ShriSathyaSai Medical College and Research institute, Sri \\ BalajiVidyapeeth Deemed to be University, Tamilnadu, India
}

*Corresponding Author: Dr. T. Rajini Samuel M.D, Assistant Professor of Biochemistry, ShriSathyaSai Medical College and Research institute, Sri BalajiVidyapeeth Deemed to be University, Tamilnadu, India

\begin{abstract}
The Non-respiratory hydrogen ion concentration was suggested as one of the measure of metabolic acid-base disorders even before many decades but clinically not applied. Recently a simple formulae was derived using standard bicarbonate for its practical application. The Non-respiratory hydrogen ion concentration is found to be inter-related in various acid-base balance theory. The three commonly used approaches for arterial blood gas interpretation are physiological, Base excess and physicochemical approach. The respiratory acid base disorders are due to the alterations in the values of $\mathrm{pCO}_{2}$ which is common in all the approaches. But for metabolic acid base disorders different parameters are used in different approaches. The physiological approach uses Bicarbonate, Base excess approach utilizes Standard base excess and in physicochemical approach Stewart's parameters like strong ion difference and the total concentration of dissociated non-volatile weak acids are applied. The aim of the current study is to derive an applied equation interrelating Stewart's theory parameters and the non-respiratory hydrogen ion concentration and its practical application in arterial blood gas interpretation.
\end{abstract}

Keywords: Non-respiratory hydrogen ion concentration, Bicarbonate, Strong ion difference

\section{INTRODUCTION}

The Non-respiratory hydrogen ion concentration was suggested as one of the measure of metabolic acid-base disorders even before many decades but clinically not applied. [I]Recently a simple formula was derived using standard bicarbonate for its practical application. The Non-respiratory hydrogen ion concentration is found to be inter-related in various acid-base balance theory.[II,III] The interdependence of the physiological, standard base excess and physicochemical approach by FenclStewart are documented in the previous studies and are important in clinical application.[IV] In arterial blood gas interpretation, respiratory acid base disorders are due to the alterations in pCO2. But for metabolic acid base disorders, different parameters are used in the 3 approaches. The interpretation by physiological approach is based on the bicarbonate-carbon dioxide buffer system, using bicarbonate as a parameter for metabolic disorders and in the Base excess approach Standard base excess or extracellular base excess ( which is the base excess at haemoglobin concentration of $5 \mathrm{~g} / \mathrm{dl}$ ) is applied.[IV,V] According to Stewarts theory, the metabolic disorders are due to primary alterations in strong ion difference(SID) and [ATOT] which reflects the plasma concentration of weak non-volatile acids namely albumin and phosphate.[IV,V,VI,VII] . Low non-respiratory hydrogen ion concentration $(\mathrm{NRH}+)$ seen in metabolic alkalosis is related to a higher value (more positive) of base excess and higher non-respiratory hydrogen ion concentration(NRH+) seen in metabolic acidos is is related to Base deficit cases.[II] In the previous research studies, the interrelationship was discussed on a theoretical basis.

The current study clearly discusses the inter-relationship between the Stewart's theory parameters and the non-respiratory hydrogen ion concentration using a derived equation.

\section{Materials And Methods}

The difference between sum of the strong cations and strong anions is called apparent strong ion difference (SIDa).Buffer base $\left(\left[\mathrm{HCO}_{3}{ }^{-}\right]+\left[\mathrm{A}^{-}\right]\right)$is numerically the same as effective strong ion difference $\left[\mathrm{SID}_{\mathrm{e}}\right]$. [IV, V, VI, VII] 
$\mathrm{SIDa}=$ [sum of the strong cations $]-$ [sum of the strong anions $]$

$\operatorname{SIDe}=\left[\mathrm{HCO}_{3}^{-}\right]+\left[\mathrm{A}^{-}\right]$

$\left[\mathrm{A}^{-}\right]$or $\left[\mathrm{A}^{-}\right.$tot $]$- denotes the total concentration of dissociated non-volatile weak acids namely albumin (Alb) and phosphate $(\mathrm{Pi})$.

Strong ion Gap (SIG):

$\mathrm{SIG}=\mathrm{SIDa}-\mathrm{SIDe}$

$\left\{\mathrm{SIDe}=\mathrm{HCO}_{3}^{-}+\mathrm{A}^{-}\right.$tot $\}$

Strong ion gap $=\mathrm{SIDa}-\mathrm{HCO}_{3}^{-}-\mathrm{A}^{-}$tot

$\mathrm{HCO}_{3}=\mathrm{SIDe}-\mathrm{A}$ tot

$\mathrm{HCO}_{3}=\mathrm{SIDa}-\mathrm{A}{ }^{-}$tot - SIG

If SIG is zero then SIDe and SIDa are equal.

Calculation of $\mathrm{NRH}^{+}$(Non-Respiratory hydrogen ion concentration)

$\mathrm{H}^{+}$(Hydrogen ion concentration $)=\left\{24 \mathrm{X} \mathrm{pCO}_{2}\right\} / \mathrm{HCO}_{3}$

The above equation is based on modified Henderson equation.[VIII]

$\mathrm{HCO}_{3}=\left\{24 \mathrm{XpCO}_{2}\right\} / \mathrm{H}^{+}$

$\mathrm{NRH}^{+}$- Hydrogen ion concentration at non-respiratory $\mathrm{pH}$ (at $\mathrm{pCO}_{2} 40 \mathrm{~mm}$ of $\mathrm{Hg}$ ).

This calculated hydrogen ion concentration equivalent of standard bicarbonate represents the "nonrespiratory hydrogen ion concentration' [NRH $\left.{ }^{+}\right]$. [II, III]

$\mathrm{NRH}^{+}=\left\{24 \mathrm{X} \mathrm{pCO}_{2}\right\} / \mathrm{Std} \mathrm{HCO}_{3}$

$$
=\{24 \times 40\} / \mathrm{Std} \mathrm{HCO}_{3}
$$

$\mathrm{NRH}^{+}=960 / \mathrm{Std} \mathrm{HCO}_{3}$

$\mathrm{Std} \mathrm{HCO}_{3}=\{24 \mathrm{X} 40\} / \mathrm{NRH}^{+}$

$\mathrm{HCO}_{3} / \mathrm{Std} \mathrm{HCO}_{3}$ :

$\mathrm{HCO}_{3} / \operatorname{Std} \mathrm{HCO}_{3}=\left\{\left(24 \mathrm{X} \mathrm{pCO}_{2}\right) \mathrm{XNRH}^{+}\right\} /\left\{\mathrm{H}^{+} \mathrm{X}(24 \mathrm{X} 40)\right\}$

$=\left\{\left(24 \mathrm{X} \mathrm{pCO}_{2}\right) \mathrm{X}\left(\mathrm{NRH}^{+} / \mathrm{H}^{+}\right)\right\} /\{24 \mathrm{X} 40\}$

$\mathrm{NRH}^{+} / \mathrm{H}^{+}$:

$\left.\left[\mathrm{NRH}^{+}\right] / \mathrm{H}^{+}\right]=\left\{(24 \mathrm{X} 40) / \operatorname{Std} \mathrm{HCO}_{3}\right\} /\left\{\left(24 \mathrm{X} \mathrm{pCO}_{2}\right) / \mathrm{HCO}_{3}\right\}$

$$
=\left\{(24 \mathrm{X} 40) \mathrm{X}\left(\mathrm{HCO}_{3} / \mathrm{Std}_{\mathrm{HCO}}\right)\right\} /\left\{24 \mathrm{X} \mathrm{pCO}_{2}\right\}
$$

Re-arranging the equation,

$\mathrm{HCO}_{3}=\operatorname{Std} \mathrm{HCO}_{3} \mathrm{X}\left\{(24 \mathrm{X} \mathrm{pCO}) \mathrm{X}\left(\mathrm{NRH}^{+} / \mathrm{H}^{+}\right)\right\} /\{24 \mathrm{X} 40\}$

Substituting the relation of bicarbonate, standard bicarbonate and $\mathrm{HCO}_{3} / \mathrm{Std}^{\mathrm{HCO}} \mathrm{H}_{3}$ in the above equation, we get,

$\mathrm{SIDa}-\mathrm{A}^{-}$tot $-\mathrm{SIG}=\left\{960 / \mathrm{NRH}^{+}\right\} \mathrm{X} \mathrm{HCO}_{3} / \mathrm{Std}_{\mathrm{HCO}}$

Where $\mathrm{HCO}_{3}=\mathrm{SIDa}-\mathrm{A}$ tot $-\mathrm{SIG}$;

Std $\mathrm{HCO}_{3}=960 / \mathrm{NRH}^{+}$;

$\left.\mathrm{HCO}_{3} / \mathrm{Std} \mathrm{HCO}_{3}=\left\{(24 \mathrm{X} \mathrm{pCO})_{2}\right)\left(\mathrm{NRH}^{+} / \mathrm{H}^{+}\right)\right\} /\{24 \mathrm{X} 40\}$

\section{Results}

250 Arterial blood gas analys is sample data's were utilized and the relationship between Bicarbonate, standard bicarbonate and the ratio Bicarbonate/Standard bicarbonate with the non- respiratory hydrogen ion concentration is graphically analysed and shown in the figures 1,2 and 3 . 


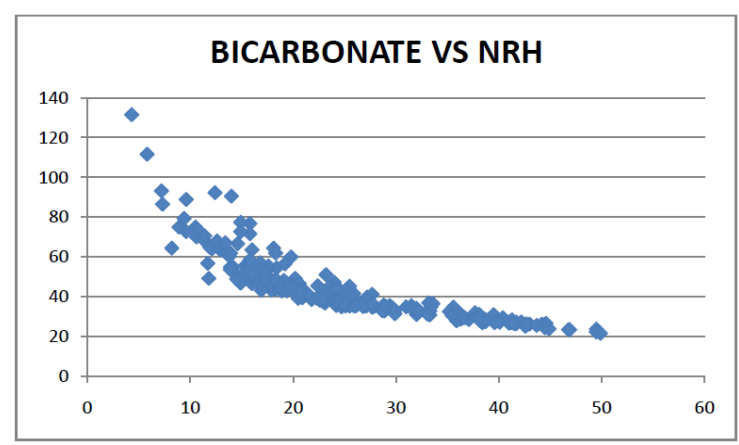

Figure1. Bicarbonate VS Non-Respiratory Hydrogen Ion Concentration ' $\left[\mathrm{NRH}^{+}\right]$.

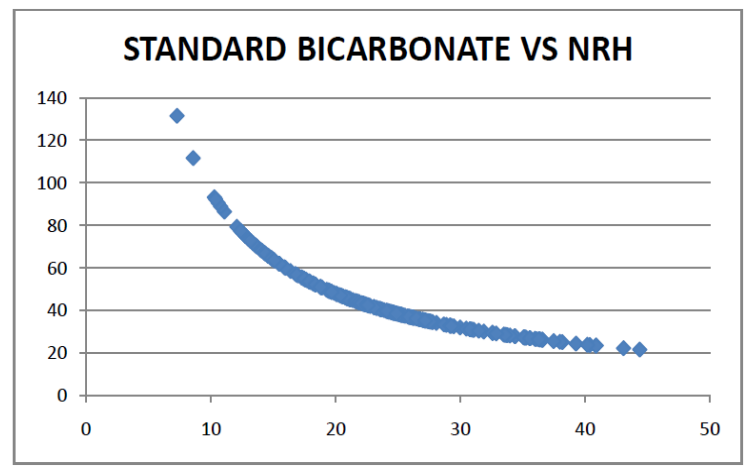

Figure2. Standard Bicarbonate VS non-respiratory hydrogen ion concentration '[NRH+].

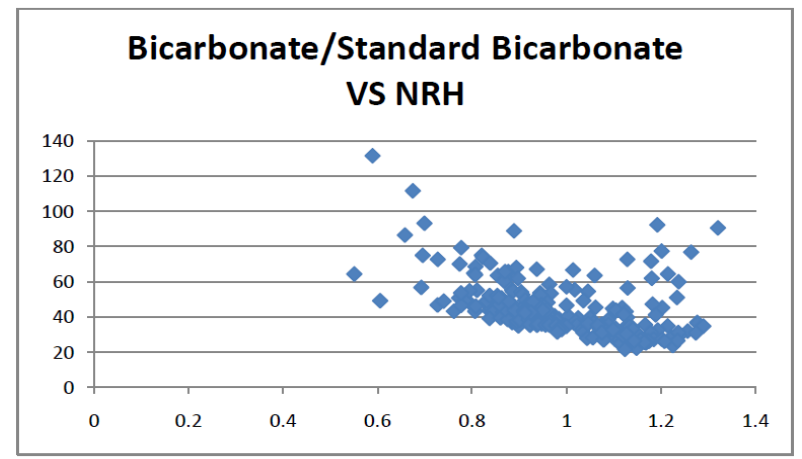

Figure3. Bicarbonate/Standard Bicarbonate VS non-respiratory hydrogen ion concentration '[NRH+].

\section{DIS CUSS ION}

Strong ions completely dissociate in solution so it is always present in a fully dissociated state. But Weak acids partially dissociate in solution so, both the un-dissociated form (HA) and the dissociated form (anionic component $\mathrm{A}^{-}$) are present in the solution. The difference between the sum of strong cations and anions is called the apparent strong ion difference (SIDa).This strong ion difference(SID) effect is balanced by the plasma buffer base (effective strong ion difference)representing sum of the plasma bicarbonate and anionic component of albumin and phosphate to preserve electrical neutrality. The normal value of SID is around $40 \mathrm{mEq} / \mathrm{L}$. [IX]

The application of water dissociation concept plays a significant part in understanding the role of strong ion difference in causing acid-base disturbances. The normal $\mathrm{pH}$ of the extracellular fluid is around 7.4, so the $\left[\mathrm{OH}^{-}\right]$is almost always greater than $\left[\mathrm{H}^{+}\right]$. The water itself presents a huge source of $\left[\mathrm{H}^{+}\right]$and the $\left[\mathrm{H}^{+}\right]$of pure water at standard temperature and pressure is actually more than double that of most bodily solutions. [IX]

Water dissociation (auto-ionization) occurs endo-thermically due to electric field fluctuations between neighbouring molecules. At $25^{0} \mathrm{c}$ pure water has a k'w of $1.008 \times 10^{-14} \mathrm{~mol}^{2} \mathrm{~L}^{-2}$. At $37^{0} \mathrm{c}$ in the body fluids, the value of $\mathrm{k}^{\prime} \mathrm{w}$ is $4.4 \times 10^{-14} \mathrm{~mol}^{2} \mathrm{~L}^{-2}$. [IX]

The ionic product of water is the product between the concentration of hydrogen and hydroxyl ions which is a constant at a given temperature. In the body fluids, the hydrogen ions does not exist as naked protons but in the form of oxonium or hydroxonium ions $\left(\mathrm{H}_{3} \mathrm{O}^{+}\right)$. (Commonly called 'hydrogen ion concentration' is related to the $\mathrm{pH}$, where $\mathrm{pH}=-\log _{10}\left(\left[\mathrm{H}_{3} \mathrm{O}^{+}\right]\right)$ 
$2 \mathrm{H}_{2} \mathrm{O} \leftrightarrow \mathrm{H}_{3} \mathrm{O}^{+}+\mathrm{OH}^{-}$

$\mathrm{K}^{\prime} \mathrm{W}=\left[\mathrm{H}_{3} \mathrm{O}^{+}\right] \mathrm{X}\left[\mathrm{OH}^{-}\right]$

The product of hydrogen ion $\left(\left[\mathrm{H}^{+}\right]\right)$concentration and the hydroxyl ion $\left(\left[\mathrm{OH}^{-}\right]\right)$concentration is a constant. So, whenever the concentration of hydrogen ion changes the hydroxyl ion concentration also changes to maintain the constant ionic product of water. If the $\left[\mathrm{H}^{+}\right]$increases, $\left[\mathrm{OH}^{-}\right]$decreases, similarly when $\left[\mathrm{H}^{+}\right]$decreases the $\left[\mathrm{OH}^{-}\right]$increases. The hydrogen ion concentration in the body fluids is in nano moles/L because the water dissociation is very little.

The strong ion difference effect has a powerful electrochemical effect on water dissociation. The water is amphoteric so it can behave both as an acid and a base. If the concentration of Chloride ion (negative anion) is increased or the concentration of sodium ion (positive cation) is decreased, the strong ion difference value is decreased so, water (behaves like an acid) dissociates more to form hydrogen ions (positive charge) to maintain electrical neutrality. So whenever SID is decreased it results formation of more hydrogen ions resulting in acidosis.

Similarly, if the concentration of chloride ion (negative anion) is decreased or the concentration of sodium ion (positive cation) is increased, the strong ion difference value is increased so, water (behaves like a base) dissociates more to form hydroxyl ions (negative charge) to maintain electrical neutrality. So increased SID results in the formation of less hydrogen ions leading to alkalosis. Sodium and chloride are the principal contributors to the strong ion difference. Dehydration and overhydration changes the strong ion difference by concentrating or diluting it respectively. [IX]

In previous research article it was mentioned that changes in strong ion difference( SID) leading to changes in hydrogen ion concentration $\left(\mathrm{H}^{+}\right)$secondary to water dissociation concept, is controversial and lacks experimental evidence. [X]But in the current research study, the application of water dissociation concept by the changes in the strong ion difference effect in causing changes in acid base disturbances is clearly explained.

The concentrations of Weak acids cause a slight increase in the hydrogen ion concentration of the plasma. Under normal conditions, this is not significant but in critically ill patients with multi-organ failure it is quiet significant. Therefore, a decrease in weak acids will cause alkalosis, and an increase produces acidosis.

At physiological $\mathrm{pH}$, the weak acids reversibly combine with protons to form the ir conjugate base. [9] $\mathrm{H}^{+}+\mathrm{A}^{-} \leftrightarrow \mathrm{HA}$

$\mathrm{A}^{-}$or $\mathrm{A}^{-}$tot: Total concentration of dissociated weak acids (anionic component only denoting the sum of the electric charge carried by them mainly albumin and phosphate)

A tot: Total concentration of weak acids including both the dissociated (anionic component $\mathrm{A}^{-}$) and un-dissociated form (HA).

The total concentration of non-volatile weak acids (A tot) is a constant ( according to law of conservation of mass) and an independent variable causing metabolic acid-base disorders but A-tot (total concentration of dissociated weak acids) is a dependent variable because it alters with changes in $\mathrm{pH}$ and $\mathrm{A}$ tot. [VI, VII, IX]

Whenever the hydrogen ion concentration increases in the blood, the weak acids combines with protons (decreased dissociation) to form the neutral conjugate base. Similarly a decrease in SID results in acidosis which causes decrease in concentration of dissociated non-volatile acids. An increase in $\mathrm{pCO} 2$ reacts with water molecules to form carbonic acid which dissociates to form $[\mathrm{H}+]$ and bicarbonate. So, increase in $\mathrm{pCO} 2$ reduces the dissociation of non-volatile weak acids.

Similarly, when the hydrogen ion concentration decreases in the blood, the equilibrium shifts resulting in more dissociation to form hydrogen ions and the dissociated weak acids. [IX] An increase in SID results in alkalos is which causes an increase in the concentration of dissociated non-volatile acids.

The changes in the strong ion difference results in electrical force that affects the weak volatile acid pCO2/carbonic acid. As the SID increases, the bicarbonate also increases, and as the SID decreases, the bicarbonate also decreases. [IX]

The relation between non-respiratory hydrogen ion concentration and the Stewart's parameters by a newly applied equation is given below.

SIDa- A tot - SIG $=\left\{960 / \mathrm{NRH}^{+}\right\} \mathrm{X} \mathrm{HCO}_{3} / \mathrm{Std}_{\mathrm{HCO}_{3}}$ 
SIDa- $\left(\mathrm{A}^{-}\right.$tot $\left.+\mathrm{SIG}\right)=\left\{960 / \mathrm{NRH}^{+}\right\} \times \mathrm{XCO}_{3} / \mathrm{Std}^{\mathrm{HCO}}{ }_{3}$

From the above equation, the following statements are inferred.

1. The concentration of Non-respiratory hydrogen ion $\left(\mathrm{NRH}^{+}\right)$depends on the difference between the Strong ion differences (SID) and the sum total of the parameters $\mathrm{A}^{-}$or $\mathrm{A}^{-}$tot (total concentration of dissoc iated weak acids) and strong ion gap (SIG).

2. The value of the parameter $\left\{\mathrm{SIDa}-\left(\mathrm{A}^{-}\right.\right.$tot $\left.\left.+\mathrm{SIG}\right)\right\}$ which denotes bicarbonate numerically is inversely related to the concentration of non-respiratory hydrogen ion and directly influenced by the ratio $\left(\mathrm{HCO}_{3} / \mathrm{Std} \mathrm{HCO}_{3}\right)$.

3. Strong ion difference is inversely proportional to the non-respiratory hydrogen ion concentration. If Strong ion difference is higher, the non-respiratory hydrogen ion concentration will be lower indicating metabolic alkalosis. Similarly, if Strong ion difference is lower, the non-respiratory hydrogen ion concentration will be higher denoting metabolic acidosis. The strong ion difference value is influenced by the ratio $\left(\mathrm{HCO}_{3} / \mathrm{Std} \mathrm{HCO}_{3}\right)$ which alters depending on the values of $\mathrm{pCO}_{2}$.

4. The total concentration of dissociated weak acids ( $\mathrm{A}^{-}$or $\mathrm{A}^{-}$tot) is directly proportional to the nonrespiratory hydrogen ion concentration. If $\mathrm{A}_{\text {tot }}$ is higher, the non-respiratory hydrogen ion concentration will be higher contributing to metabolic acidosis. Similarly, if $\mathrm{A}^{-}$tot is lower, the non-respiratory hydrogen ion concentration will be lower contributing to metabolic alkalosis.

5. The Strong ion gap (SIG) is directly proportional to the non-respiratory hydrogen ion concentration. If SIG is higher, the non-respiratory hydrogen ion concentration will be higher contributing to metabolic acidosis.

The graphical relationship between bicarbonate, standard bicarbonate and their ratio with the parameter non-respiratory hydrogen ion concentration is clearly depicted in the graphs 1, 2 and 3 respectively. It is very clear that bicarbonate is highly influenced by the alterations of $\mathrm{pCO}_{2}$ values. From the newly derived relation, the following are clearly observed. The decrease in strong ion difference results in low Bicarbonate value indicating metabolic acidosis. If the strong ion difference is decreased with increase in unmeasured ions, then the bicarbonate value will be very low indicating severe metabolic acidos is. The increased presence of unmeasured ions will not be clearly noticed if the concentration of dissociated weak acid (Atot) is very low, because there is no net changes in the sum total of the parameters (A tot + SIG) resulting in a normal bicarbonate value. The presence of unmeasured ions is masked due to the alkalizing effect of decreased (Atot). The increase in strong ion difference results in high $\mathrm{B}$ icarbonate value indicating metabolic alkalosis. If the values of strong ion difference and the strong ion gap are normal but $\mathrm{A}^{-}$tot parameter is highly decreased then it results in high bicarbonate value denoting metabolic alkalosis. This applied equation helps in understanding the relationship between these various parameters used for the interpretation of metabolic acid-base disorders.

\section{CONCLUSiON}

The newly derived equation interrelating the Stewart's parameters with the non-respiratory hydrogen ion concentration and the ratio between bicarbonate and standard bicarbonate denoting the respiratory influence may help in better understanding and correlating in the interpretation of metabolic acid base disorders under different conditions.

\section{REFERENCES}

[1] J. T. SueroThe Usefulness of Non-Respiratory Hydrogen Ion Concentration and Its Relationship to the Traditional Acid-Base Parameters Clin. Biochem, 1969; 2: 177-185

[2] Rajini Samuel T Application and Inter-Relationship of Non-Respiratory Hydrogen Ion Concentration in Acid-Base Balance Theory International Journal of Clinical Chemistry and Laboratory Medicine (IJCCLM), 2018; 4(3): 1-13.

[3] Rajini Samuel A Graphical Representation For Aiding Arterial Blood Gas Interpretation Using NonRespiratory And Respiratory pHWJPMR, 2018,4(12), 192-202

[4] Horacio J. Adrogue ,F. John Gennari, John H. Galla and Nicolaos E. Madias Assessing acid-base disorders Kidney International (2009) 76, 1239-1247

[5] Magder S, Emami A. Practical approach to physical-chemical acid-base management. Stewart at the bedside. Ann Am Thorac Soc. 2015;12:111-117 
[6] Howard E. Corey Stewart and beyond: New models of acid-base balance Kidney International, Vol. 64 (2003), pp. 777-787

[7] Howard E Corey Bench-to-bedside review: Fundamental principles of acid-base physiology Crit Care. 2005; 9(2): 184-192.

[8] Rajini Samuel, Ilanchezian, BalajiRajagopalan Application of Modified Henderson Equation In ABG Interpretation Int. J. Pharm. Sci. Rev. Res, 2016; 37(2): 169-177

[9] GunjanChawla, Gordon Drummond Water, strong ions, and weak ions Continuing Education in Anaes thesia, Critical Care \& Pain 2008; 8(3): 108-112

[10] Fabio D Masevicius, ArnaldoDubinHas Stewart approach improved our ability to diagnose acid-base disorders in critically ill patients? World J Crit Care Med 2015; 4(1): 62-70

Citation: Dr. T. Rajini Samuel M.D "An Applied Equation Inter-Relating Stewart's Parameter and NonRespiratory Hydrogen Ion Concentration in Arterial Blood Gas", International Journal of Clinical Chemistry and Laboratory Medicine (IJCCLM), vol. 5, no.1, pp. 23-28, 2019. http://dx.doi.org/10.20431/24557153.0501004

Copyright: (C) 2019 Authors. This is an open-access article distributed under the terms of the Creative Commons Attribution License, which permits unrestricted use, distribution, and reproduction in any medium, provided the original author and source are credited. 